\title{
Ultrafast Coherent Spectroscopy of the Fermi Edge Singularity
}

\author{
Diego Porras, J. Fernández-Rossier and C. Tejedor \\ Departamento de Física Teórica de la Materia Condensada. Universidad Autónoma de Madrid. \\ Cantoblanco, 28049 Madrid. Spain
}

\begin{abstract}
In this work we present a theoretical description of the transient response of the Fermi Edge Singularity (FES). We study the linear and the nonlinear response of an n-doped QW to laser pulses in the Coherent Control (CC) and Four Wave Mixing (FWM) Configurations. By means of a bosonization formalism we calculate the FWM signal emitted by the sample when it is excited by pulses spectrally peaked around the FES and we show that the long time behavior of the nonlinear signal is very similar to the linear case.
\end{abstract}

Keywords: Fermi Edge Singularity, Coherent Control, Four Wave Mixing, Bosonization, GaAs Quantum Wells.

\section{Motivation}

The promotion of an electron from a localized state in a valence band (VB) to an empty state in a partially filled conduction band (CB) is accompanied by a dynamical response of the Fermi gas. The enhancement of the absorption probability when the new electron is promoted just above the Fermi energy is known as the Fermi Edge Singularity (FES). This phenomena has been experimentally observed, by means of continuous wave spectroscopy, both in metal $\$$ and doped semiconductors 1 . FES is understood as a consequence of the appearance of a coherent gas of low energy collective excitations of the Fermi sea due to both the sudden appearance of the localized hole and the new electron. It has been shown 3 that these collective excitations can be considered as Tomonaga bosons which are in a Glauber coherent state.

In this paper we propose two types of transient coherent experiments, Coherent Control (CC) and Four Wave Mixing (FWM) in doped Quantum Wells, in order to address the following questions: i) Can be these Tomonaga bosons coherently controlled? ii) How is the decay of the Tomonaga bosons coherence? and iii) What is the nonlinear response of the FES ? In this context, pump and probe experiments have been suggested for the transient spectroscopy of the FESE. The experience acquired in the case of undoped samples shows that both CC $\mathrm{O}_{\text {and }} \mathrm{FWM}$ are precise and conceptually simple tools to probe excitonic coherence and nonlinearity. We propose to excite an n-doped QW with laser pulses which promote electrons from localized states in the VB to the Fermi level in the CB. The spectral width of the laser pulses $(1 / \Delta t)$ must be much smaller than the Fermi energy (measured with respect the bottom of the $\mathrm{CB}), \epsilon_{F}$, so that the only intraband excitations are the Tomonaga bosons. Therefore, our theoretical approach is not valid to address the FWM experiment of Kim et al. $\mathrm{Q}$ but it could be compared to the zero magnetic field case of the experiments of Bar-Ad et al. 目. 


\section{Hamiltonian and Linear Response: Coherent Control}

The problem of the linear response function of the FES was solved long time ago in a particularly simple way by a description of the Fermi Sea excitations in terms of bosonic operators. If we consider that the photoexcited hole creates a spherical localized potential then the Hamiltonian is:

$$
H=E_{0} d^{\dagger} d+\sum_{k=0}^{k_{D}} \epsilon_{k} a_{k}^{\dagger} a_{k}+\frac{V}{N} \sum_{k, k^{\prime}=0}^{k_{D}} a_{k}^{\dagger} a_{k^{\prime}} d^{\dagger} d
$$

where $E_{0}, d^{\dagger}$ are the energy and creation operator of the localized hole, $a_{k}^{\dagger}$ are the creation operators of the electrons in the conduction band and $k_{D}$ is the bandwidth 3 . Here we will only consider s-wave scattering, and reduce the initial problem to a 1-D one, so that the index $\mathrm{k}$ corresponds to the radial wave function index. The polarization induced in the sample by an electric field is described by the operator $P(t)=\mu a^{\dagger} d^{\dagger}+h$.c., where $a=\sum_{k=0}^{k_{D}} a_{k}$ and $\mu$ is the dipole matrix element. The mean value of $\mathrm{P}(\mathrm{t})$ in the absence of inelastic scattering (decoherence) can be calculated by means of the linear response function. For zero temperature $(T=0)$ we have:

$$
\left.\chi^{(1}\right)(t) \propto-i \mu^{2} \theta(t)\left(i \epsilon_{F} t\right)^{\alpha} e^{-i \epsilon t}+\text { h.c. }
$$

where $\rho$ is the density of states at the Fermi surface, $\epsilon=E_{g}+\epsilon_{F}-(V \rho)^{2} \epsilon_{F}$ is the FES resonance energy, $E_{g}$ is the semiconductor gap and $\alpha=(1+V \rho)^{2}$ is the exponent of the singularity. Let us consider now the CC of the linear response of the sample. The signal emitted is proportional to the polarization created by the incident laser. We will work in the Rotating Wave Approximation (RWA). If the laser pulse has the form $E(t)=E_{A}(t)+E_{B}(t)+h . c$., with $E_{A}(t)=\mathcal{E}_{A}(t) e^{-i \epsilon t}, E_{B}(t)=E_{A}(t-\tau)$, and we approximate $\mathcal{E}_{A}(t)$ by a delta function, then this signal is (for $t>>\tau$ ):

$$
F^{(1)} \propto-i\left(i \epsilon_{F} t\right)^{\alpha}\left(1+e^{i \epsilon \tau}\right)+h . c
$$

This quantity can be measured by means of the time integrated luminescence, as in the undoped case 5 , and it turns out to be $I \propto\left|F^{(1)}\right|^{2} \propto 1+\operatorname{Cos}(\epsilon \tau)$. Therefore, CC of the FES is possible. This can be understood as the interference of the electric dipole induced by the first pulse with that induced by the second pulse. Microscopically, Tomonaga bosons are being coherently controlled in much the same way than the CC of optical phonons $巴$.

\section{Four Wave Mixing signal}

In FWM experiments, the system is excited by two phase locked pulses having momenta $\mathbf{k}_{\mathbf{A}}$ and $\mathbf{k}_{\mathbf{B}}$ with $\left|\mathbf{k}_{\mathbf{A}}\right|=\left|\mathbf{k}_{\mathbf{B}}\right|$ and a relative delay $\tau$. The excitation of electrons near the Fermi surface can be achieved by choosing pulses spectrally peaked around the FES resonance. The FWM signal comes from the interference between the polarization created by the first pulse $(t=0)$ and that of the second pulse $(t=\tau)$. We neglect the (exponential) decay of the polarization due to inelastic scattering and to inhomogeneous broadening of the holes. We show that there is an intrinsic potential decay, due to the dephasing of the Tomonaga Bosons, which could be observed in favorable conditions. 
To calculate the intrinsic decay we need to obtain the third-order contribution to the polarization induced in the sample, which is proportional to the FWM signal emitted in the $2 \mathbf{k}_{\mathbf{B}}-\mathbf{k}_{\mathbf{A}}$ direction. We consider two pulses $E_{A, B}(t)=\mathcal{E}_{A, B}(t) e^{-i \epsilon t}$, where $\mathcal{E}_{A, B}(t)$ are the amplitudes of the pulses in the directions $\mathbf{k}_{\mathbf{A}}, \mathbf{k}_{\mathbf{B}}$. The FWM signal can be written in terms of the third-order response function:

$$
F(t)=\int_{-\infty}^{t} d t_{1} d t_{2} d t_{3} \chi^{(3)}\left(t-t_{1}, t-t_{2}, t-t_{3}\right) E_{A}^{*}\left(t_{2}\right) E_{B}\left(t_{1}\right) E_{B}\left(t_{3}\right)
$$

By extending up to third order the usual perturbation scheme developed for obtaining the Kubo formula, one obtains:

$$
\begin{aligned}
\chi^{(3)}(t)=-i[\theta( & \left.-t_{1}\right) \theta\left(t_{1}-t_{2}\right) \theta\left(t_{2}-t_{3}\right)\left\langle P(t) P\left(t_{1}\right) P\left(t_{2}\right) P\left(t_{3}\right)\right\rangle \\
& \left.+\theta\left(t-t_{1}\right) \theta\left(t_{1}-t_{2}\right) \theta\left(t_{2}-t_{3}\right)\left\langle P\left(t_{2}\right) P\left(t_{1}\right) P(t) P\left(t_{3}\right)\right\rangle\right]+ \text { h.c. }
\end{aligned}
$$

where the brackets \langle\rangle mean thermal averages. Equation (5) has been obtained within the RWA in which the width $\Delta t$ of each pulse is much larger than the period $\propto \epsilon^{-1}$ of the exciting light. Here we will also consider, for simplicity, delta pulses: $E_{A}(t)=E_{A} \delta(t) e^{-i \epsilon t}$, $E_{B}(t)=E_{B} \delta(t-\tau) e^{-i \epsilon(t-\tau)}$. This assumption does not imply a contradiction with the RWA: it is justified if the variations of the correlation functions are slow enough in a time interval $\Delta t$. In (5) only the second term takes a non-vanishing value. Our main task now is the calculation of the correlation function, for which the bosonization formalism is extremely useful. If we define $H_{i}, H_{f}$ as the Hamiltonian without and with hole, respectively, then:

$$
\left\langle P\left(t_{2}\right) P\left(t_{1}\right) P(t) P\left(t_{3}\right)\right\rangle=\mu^{4}\left\langle e^{i H_{i} t_{2}} a e^{-i H_{f}\left(t_{2}-t_{1}\right)} a^{\dagger} e^{-i H_{i}\left(t_{1}-t\right)} a e^{-i H_{f}\left(t-t_{3}\right)} a^{\dagger} e^{-i H_{i} t_{3}}\right\rangle
$$

If we assume that $\Delta t>>\left(\epsilon_{F}\right)^{-1}$, only excitations near the Fermi Edge will be relevant and we can use the Schotte ${ }^{3}$ approach to reexpress this correlation function in the bosonization formalism:

$$
\left\langle P\left(t_{2}\right) P\left(t_{1}\right) P(t) P\left(t_{3}\right)\right\rangle \propto\left\langle B^{\dagger}(t) B\left(t_{1}\right) B^{\dagger}\left(t_{2}\right) B\left(t_{3}\right)\right\rangle e^{-i \epsilon t} e^{i \epsilon\left(t_{1}-t_{2}+t_{3}\right)}
$$

where $B^{\dagger}$ are operators that create a coherent state of Tomonaga bosons $b_{k}^{\dagger}$ :

$$
B(t)=\exp \left[\sum_{k=0}^{k_{F}} \frac{(1+V \rho)}{\sqrt{k N}}\left(b_{k}^{\dagger} e^{i \frac{k}{\rho} t}-b_{k} e^{-i \frac{k}{\rho} t}\right)\right]
$$

After integration with the pulses we arrive at:

$$
F^{(3)}(t) \propto-i \mu^{4} e^{-i \epsilon(t-2 \tau)} \theta(t-\tau) \theta(\tau)\left\langle B^{\dagger}(0) B(\tau) B^{\dagger}(t) B(\tau)\right\rangle
$$

We consider a bath of bosons in equilibrium at temperature $\mathrm{T}$ (with $k_{B} T<\epsilon_{F}$ ) for the thermal average:

$$
\begin{aligned}
F^{(3)}(t) \propto-i \mu^{4} \theta(t-\tau) \theta(\tau) e^{-i \epsilon(t-2 \tau)} \exp & \left(\alpha \sum_{k=0}^{k_{F}}\left(\frac{1}{2}+N_{B}\right) \frac{1}{k N}\left|1-2 e^{i \frac{k}{\rho} \tau}+e^{i \frac{k}{\rho} t}\right|^{2}\right) \\
& \times \exp \left(i \alpha \sum_{k=0}^{k_{F}} \frac{1}{k N}\left(-2 \sin \frac{k}{\rho} \tau+\sin \frac{k}{\rho} t\right)\right)
\end{aligned}
$$


where $N_{B}=\left(e^{\frac{k / \rho}{k_{B} T}}-1\right)^{-1}$. We can express the sum in momentum space as an integral: $\sum_{k=0}^{k_{F}}=\int_{0}^{k_{F}} d k$. If we consider the limit $t>>\tau$ we obtain a very simple expression for the complete FWM signal:

$$
F^{(3)}(t, T) \propto-i \mu^{4} \theta(t-\tau) \theta(\tau) e^{-i \epsilon(t-2 \tau)}\left[\left(\frac{i \epsilon_{F}}{\pi k_{B} T}\right)^{3} \sinh ^{2}\left(\pi k_{B} T \tau\right) \sinh \left(\pi k_{B} T t\right)\right]^{\alpha}
$$

This signal has the zero temperature limit:

$$
F^{(3)}(t, T=0) \propto-i \mu^{4} \theta(t-\tau) \theta(\tau) e^{-i \epsilon(t-2 \tau)}\left(i \epsilon_{F} \tau\right)^{2 \alpha}\left(i \epsilon_{F} t\right)^{\alpha}
$$

\section{Conclusions}

In this paper we have shown that temporal CC of the Tomonaga bosons can be achieved. We have also shown that the bosonization formalism is a simple tool to calculate the nonlinear susceptibility $\chi^{(3)}$, for both zero and nonzero temperature. We have calculated the FWM signal, for the case of Delta pulses, and we have shown that $F^{(1)}$ and $F^{(3)}$ have an identical dependence on the detection time $t$ for both $T=0$ and $T \neq 0$. Neglecting inelastic scattering $F^{(3)}$ has an intrinsic potential decay as a function of $\tau$. It must be pointed out that this potential decay will be superimposed, in real experiments, to an exponential decay produced by electron-electron, electron-phonon interactions and inhomogeneous broadening of the holes.

\section{Acknowledgements}

Work supported in part by MEC of Spain under contract PB96-0085, Fundacion Ramón Areces and CAM under contract 07N/0026/1998. Diego Porras thanks Spanish Education Ministry for its FPU grant.

\section{References}

1. G.D. Mahan, Many-Particle Physics, Ed. Plenum, New York, 1981

2. M. S. Skolnik et al., Phys. Rev. Lett 58, 2130 (1987)

3. K. D. Schotte and U. Schotte, Phys. Rev., 182, 479 (1969).

4. I. Brener, W.H. Knox and W. Schaefer, Phys. Rev. B 51, 2005 (1995), I.Perakis et al., J. Optc. Soc. Am. B13 1313 (1996)

5. A.P. Heberle, J.J. Baumberg, K. Köhler, , Phys. Rev. Lett. 75, 2598 (1995). X. Marie et al., Phys. Rev. Lett. 79, 3222, (1997). J.Fernandez-Rossier, C. Tejedor and R. Merlin, J. of Phys.Cond. Mat., in press.

6. S. Weiss et al., Phys. Rev. Lett. 69, 2685 (1992)

7. D. S. Kim et al., Phys. Rev. Lett., 68, 2838 (1992).

8. S. Bar-Ad et al. Phys. Rev. Lett. 72, 776 (1994)

9. See, for instance, K. Ohtaka and Y. Tanabe, Rev. Mod. Phys., 62, 929 (1990).

10. M. U. Wehner, Phys. Rev. Lett., 80, 1992 (1998) 